\title{
Efeito comparativo entre a infiltração de plasma rico em plaquetas e o uso de corticosteroides no tratamento de osteoartrite do joelho: Estudo clínico prospectivo e randomizado*
}

\section{Comparative Effect between Infiltration of Platelet-rich Plasma and the Use of Corticosteroids in the Treatment of Knee Osteoarthritis: A Prospective and Randomized Clinical Trial}

\author{
Marianna Ribeiro de Menezes Freire ${ }^{1}$ Philippe Mota Coutinho da Silva ${ }^{1}$ Arthur Rangel Azevedo ${ }^{2}$ \\ Denison Santos Silva ${ }^{3}$ Ronald Bispo Barreto da Silva ${ }^{3}$ Juliana Cordeiro Cardoso ${ }^{3}$ \\ ${ }^{1}$ Faculdade de Medicina, Universidade Tiradentes (Unitmed), Aracaju, \\ SE, Brasil \\ 2 Instituto de Assistência Médica ao Servidor Público Estadual de São \\ Endereço para correspondência Marianna Ribeiro de Menezes Freire, \\ Faculdade de Medicina, Universidade Tiradentes (Unitmed), \\ Paulo (lamspe), São Paulo, SP, Brasil \\ 3 Universidade Tiradentes (Unitmed), Aracaju, SE, Brasil
}

Rev Bras Ortop 2020;55(5):551-556.

\section{Resumo}

Palavras-chave

- osteoartrite

- corticoterapia

- plasma rico em plaquetas
Objetivo Analisar a eficácia do uso de plasma rico em plaquetas, obtido do sangue periférico e autólogo dos pacientes, na redução das queixas álgicas e melhoria funcional dos pacientes portadores de osteoartrite de joelhos, em comparação com o tratamento padrão com injeção de corticosteroides de depósito, como a triancilonola.

Métodos Os pacientes foram acompanhados clinicamente na consulta pré-infiltrativa, com avaliação quantitativa através das escalas Knee Society Score (KSS), Western Ontario and McMaster Universities Osteoarthritis Index (WOMAC), e Kellgren and Lawrence. Além disso, os pacientes foram reavaliados com as mesmas escalas após 1 mês e 6 meses de intervenção com $2,5 \mathrm{~mL}$ de acetato de triancinolona ou $5 \mathrm{~mL}$ de plasma rico em plaquetas. O estudo foi feito em 50 pacientes portadores de osteoartrite de joelhos atendidos no Centro de Especialidades Médicas, divididos em amostras equivalentes randomizadas para cada terapia.

Resultados No presente estudo, verificaram-se redução dos valores em escalas álgicas, como a WOMAC, e elevação das pontuações em escalas funcionais, como a KSS, evidenciadas em 180 dias após o uso de plasma rico em plaquetas, uma terapia feita a partir do sangue autólogo do paciente e com menos efeitos colaterais.
* Trabalho desenvolvido no Centro de Especialidades Médicas da Universidade Tiradentes (Unitmed), Aracaju, SE, Brasil. Publicado Originalmente por Elsevier Editora Ltda. recebido

20 de Outubro de 2017

aceito

04 de Janeiro de 2018
DOI https://doi.org/

10.1016/j.rbo.2018.01.001. ISSN 0102-3616.
Copyright $\odot 2020$ by Sociedade Brasileira License terms de Ortopedia e Traumatologia. Published by Thieme Revnter Publicações Ltda, Rio de Janeiro, Brazil 
Abstract

\section{Keywords}

- osteoarthritis

- corticosteroids

- platelet-rich plasma
Conclusão Embora ambas as terapias com plasma rico em plaquetas e corticosteroides tenham se mostrado eficazes na redução das queixas álgicas e na recuperação funcional, houve diferença significativa entre as terapias aos 180 dias. De acordo com os resultados obtidos, no tratamento da osteoartrite do joelho, o plasma rico em plaquetas demonstrou efeitos mais duradouros em 180 dias.

Objectives The objective of the present study was to analyze the efficacy of plateletrich plasma, obtained from the peripheral and autologous blood of the patients, in the reduction of pain complaints and functional improvement of osteoarthritis of the knees, compared with the standard treatment with corticosteroid injection, such as triamcinolone.

Methods The patients were followed up clinically at the preinfiltrative visit, with quantitative evaluation using the Knee Society Score (KSS), the Western Ontario and McMaster Universities Osteoarthritis Index (WOMAC) score, and the Kellgren and Lawrence scales. In addition, they were re-evaluated with the same scales after 1 month and 6 months of intervention with $2.5 \mathrm{~mL}$ of triamcinolone acetate or $5 \mathrm{~mL}$ of plateletrich plasma. The study was performed on 50 patients with knee osteoarthritis treated at the Medical Specialty Center, divided into randomized equivalent samples for each therapy.

Results The present study verified the reduction of pain scores, such as the WOMAC score, and elevations of functional scales, such as the KSS, evidenced in 180 days when using platelet- rich plasma, a therapy that uses the autologous blood of the patient and has fewer side effects.

Conclusion Although both platelet-rich plasma and corticosteroid therapies have been shown to be effective in the reduction of pain complaints and functional recovery, there was a statistically significant difference between the therapies at 180 days. According to the results obtained, platelet-rich plasma presented longer-lasting effects within 180 days in the treatment of osteoarthritis.

\section{Introdução}

A osteoartrite é a doença articular mais comumente encontrada nas populações ocidentais e caracteriza-se por afetar principalmente os quadris, joelhos, mãos, e pés. ${ }^{1,2}$ É responsável pela incapacidade funcional progressiva e pela presença de diversos fatores etiológicos, como idade, obesidade, e fatores ocupacionais e genéticos, contribuintes para seu surgimento. $^{3}$

Segundo estudos epidemiológicos em todo o mundo, a prevalência da osteoartrite é positivamente associada com o aumento da idade e com o sexo feminino, numa proporção de 5,3 mulheres para cada 3,6 homens acometidos. ${ }^{4,5}$

A perda da homeostasia entre a síntese e degradação da matriz cartilaginosa constitui a base fisiopatológica da osteoartrite. ${ }^{6}$ Nesses pacientes, os condrócitos e as células sinoviais produzem níveis elevados de citocinas pró-inflamatórias, como a IL-11 e o TNF-a, que, por sua vez, estimulam metaloproteinases, outras interleucinas (IL-6, IL- 8), prostaglandina E2 e óxido nítrico, também estimulado por estresse mecânico. ${ }^{7}$

A progressão das lesões condrais articulares seria a principal causa de dor noturna, rigidez, edema e comprometimento da mobilidade articular, afeta negativamente a qualidade de vida, o bem-estar emocional do paciente (especialmente os idosos) e o estado geral de saúde. ${ }^{8}$

Os joelhos são as grandes articulações mais comumente afetadas, resulta em sintomas incapacitantes que comprometem a qualidade de vida em aproximadamente $10 \%$ das pessoas com mais de 55 anos, um quarto delas severamente acometidas pela imobilidade. , $^{8,9}$

Diversos medicamentos orais para alívio da dor estão disponíveis e são considerados eficazes nas fases precoces da doença. Entretanto, o tratamento sempre deve ser multifatorial, uma vez que a prescrição farmacológica isolada não é suficiente para o controle desejado da osteoartrite., ${ }^{1,9}$

Os tratamentos através de procedimentos de infiltração são normalmente reservados para pacientes com resposta insatisfatória aos regimes orais. ${ }^{10}$ Infiltrações com ácido hialurônico foram usadas por décadas, porém seu uso tem sido desencorajado devido ao alto risco de efeitos adversos e benefício irrelevante quando comparadas com as injeções intra-articulares de corticosteroides. ${ }^{11,12}$ Dessa maneira, os corticosteroides injetáveis são amplamente usados no tratamento sintomático da osteoartrite de joelhos, entretanto a sua eficácia parece ser limitada a um mês. ${ }^{12}$ 
O uso de infiltado de plasma rico em plaquetas (PRP) é considerado um tratamento regenerativo emergente para a degeneração causada pela osteoartrite, uma vez que a degranulação de plaquetas leva à liberação de diversos fatores de crescimento e citocinas que desempenham um papel crucial na homeostasia articular. ${ }^{13}$ Assim, o uso de PRP deve promover a quimiotaxia celular, síntese de matriz e proliferação da cartilagem. ${ }^{14}$

Recentemente, PRP passou a ser usado para o tratamento de osteoartrite de joelhos, entretanto as evidências clínicas ainda não são claras devido à falta de estudos padronizados quanto ao intervalo, volume e à frequência de infiltração do plasma. $^{15,16}$

O objetivo do presente estudo foi analisar a eficácia do uso de PRP, obtido do sangue periférico e autólogo dos pacientes, na redução das queixas álgicas e melhoria funcional dos pacientes portadores de osteoartrite de joelhos, em comparação com o tratamento padrão com injeção de corticosteroides de depósito, como a triancilonola.

\section{Material e Métodos}

O estudo é de caráter randomizado, controlado, longitudinal, duplo cego, comparativo, descritivo e analítico. Foi feito com pacientes portadores de osteoartrite de joelhos atendidos no Centro de Especialidades Médicas Unit (Unitmed). Os pacientes foram organizados por randomização em bloco, cuja única especificação foi a quantidade equivalente entre os pacientes, e designados para receber o tratamento com plasma rico em plaquetas, compuseram o grupo intervenção, ou receber injeção de corticosteroides, compuseram o grupo controle.

O número de componentes da amostra foi estimulado para que tenha intervalo de confiança de $95 \%$, poder de $80 \%$ e diferença entre grupos de $20 \%$; assim sendo, chegou-se a um valor de 25 pessoas no grupo intervenção e 25 no grupo controle, totalizando 50 indivíduos. Para manter a característica de duplo cego, sangue periférico foi colhido de todos os pacientes.

Para obtenção do PRP, foi usado o protocolo de dupla centrifugação descrito inicialmente por Sonnleitner et al. ${ }^{17} \mathrm{e}$ validado por Messora et al. ${ }^{18}$ Nesse protocolo, foram coletados $15 \mathrm{ml}$ de sangue periférico em tubos a vácuo de $5 \mathrm{ml}$ com citrato de sódio a $3,2 \%$. Esse material foi enviado para primeira centrifugação e a coluna superior foi então aspirada e novamente centrifugada, obtiveram-se o plasma pobre em plaqueta (PPP) e o botão eritroplaquetário. Do volume obtido, $2 / 3$ do PPP foram descartados e o restante foi usado para suspender o botão eritroplaquetário, formou o PRP em $5 \mathrm{ml}$ para solução injetável.

$\mathrm{O}$ corticosteroide usado foi o acetato de triancinolona (Triancil, Apsen Farmacêutica, São Paulo, SP, Brasil). A iinfiltração foi feita com a administração intra-articular de 2,5 ml do corticoide, na concentração de $20 \mathrm{mg} / \mathrm{ml}$, sem agente diluidor.

Ambas as infiltrações foram feitas na região intra-articular superolateral do joelho operado após botão anestésico com $2 \mathrm{ml}$ de lidocaína a $2 \%$, sem vasoconstritor. Após a injeção, os pacientes permaneceram em decúbito dorsal sem movimentação do membro injetado por 5 minutos.
Após conferir qual tratamento seria feito para o paciente, de acordo com a lista de randomização em bloco previamente gerada, um dos pesquisadores fez o envelopamento da seringa com esparadrapo opaco. Essa seringa foi então entregue para 0 médico responsável pelas infiltrações, manteve-se o mascaramento da intervenção para o paciente e para o médico.

As variáveis analisadas em cada grupo foram as seguintes: idade, gênero, idade média de diagnóstico, hipertensão, obesidade, diabetes, e cirurgia ortopédica prévia em joelho analisado.

Os critérios de inclusão foram: idade entre 30 e 90 anos, presença de osteoartrite graus II a IV, segundo a escala de Kellgreen e Lawrence, ${ }^{19}$ ausência de demais doenças inflamatórias reumáticas, ausência de tratamento anterior com corticoide IV, injetável ou oral nos últimos 12 meses e assinatura do termo de consentimento livre e esclarecido.

Os critérios de exclusão foram: doença que impossibilitasse seguimento, perda de seguimento, uso de corticoide oral ou IV durante o período de seguimento e hemoglobina menor do que $11 \mathrm{~g} / \mathrm{dl}$, contagem de plaquetas menor do que $150.000 / \mathrm{mm}^{3}$.

Para avaliar resposta terapêutica foram usados os questionários Knee Society Score (KSS) e Western Ontario and McMaster Universities Osteoarthritis Index (WOMAC), validados e padronizados para a língua portuguesa. ${ }^{20,21} \mathrm{O}$ primeiro foi a versão traduzida do $\mathrm{KSS}^{20}$, que consiste num questionário que combina informações subjetivas e objetivas sobre o aspecto funcional do paciente, como a habilidade de caminhar, subir e descer escadas, e as características associadas ao joelho, como dor, estabilidade e arco de movimentação. O KSS avalia o quadro clínico do paciente quanto à dor e ao grau de comprometimento da mobilidade e estabilidade em diversos planos. Assim, quanto maior a pontuação no KSS, mais clinicamente relevante foi a resposta terapêutica. ${ }^{22} \mathrm{O}$ segundo questionário foi o Womac, que contém 17 questões em relação ao grau de dificuldade para fazer atividades da vida diária, a fim de avaliar a funcionalidade do paciente, dor e rigidez. Quanto maior o escore, pior a função. ${ }^{21,23}$ Nesse questionário, foi usado como critério de diferença clínica relevante uma possível redução de $16 \%$ do valor total do WOMAC, feita antes da intervenção. ${ }^{24}$

Os pacientes foram avaliados antes do procedimento, após 1 mês e após 6 meses do ato infiltrativo com esses questionários. A classificação de Kellgren and Lawrence $(\mathrm{KL})^{19}$ foi usada em avaliação pré-infiltrativa por ortopedista e reumatologista experiente.

Essas informações alimentaram um banco de dados construído no software SPSS for Windows, versão 21.0 (IBM Corp., Armonk, NY, EUA). As características demográficas foram apresentadas em frequências, percentuais e respectivos intervalos de confiança de 95\% (ICs95\%) para as variáveis categóricas e em média e desvio-padrão ou mediana com percentis 25 e 75 para as variáveis contínuas.

A comparação entre médias foi feita através de métodos paramétricos (testes $t$ de Student e análise de variância [ANOVA]) ou não para- métricos (Kruskal-Wallis ou MannWhitney) para variáveis contínuas e $\mathrm{x}^{2}$ (qui-quadrado) para variáveis categóricas, conforme o tipo de distribuição assumida pela variável. Para análise dos fatores associados à variável desfecho, foram determinadas as prevalências com suas respectivas razões. Em seguida usou-se um modelo 
logístico para determinação das razões de chances (odds ratio) simples e ajustadas. Foi usado o teste de comparação múltipla de Tukey para a avaliação entre as terapias nos períodos selecionados.

O nível de significância estatística considerado, para todos os testes, foi $p=0,05$, em análises univariadas. A pesquisa assegura a confidencialidade e a privacidade dos profissionais participantes, garante o não uso das informações do questionário em prejuízo das pessoas envolvidas. 0 projeto foi aprovado pelo comitê de ética, seguiu as recomendações orientadas.

\section{Resultados}

Os 2 grupos estudados apresentaram quantitativo semelhante após a distribuição randômica, ficaram alocados 25 pacientes em cada um dos grupos. Após a inclusão no estudo, não houve perda de seguimento ou exclusão de pacientes.

Na análise demográfica da população estudada foi encontrada uma média de $64,15( \pm 8,02)$ anos no grupo de pacientes cuja terapêutica foi o PRP e de 60,21 $( \pm 5,92)$ anos no grupo de pacientes cuja terapêutica foi a infiltração com corticosteroides $(p=0,01)$. Dentre os indivíduos avaliados, a frequência de pessoas do sexo feminino foi de $84 \%(n=42)$.

No presente estudo, $90 \%$ dos pacientes encontravam-se entre o grau II e III da escala de KL, a distribuição entre os grupos está detalhada na tabela 1.

Com relação às comorbidades, pelos resultados encontrados na tabela 2, pode-se dizer que não há associação entre as comorbidades e a terapia escolhida de acordo com a seleção dos grupos.

Tabela 1 Proporção de pacientes avaliados pela escala de Kellgren e Lawrence de acordo com a terapia designada

\begin{tabular}{|l|l|l|}
\hline $\begin{array}{l}\text { Kellgren e } \\
\text { Lawrence }\end{array}$ & $\begin{array}{l}\text { Plasma rico em } \\
\text { plaquetas (\%) }\end{array}$ & Corticosteroide \\
\hline I & - & $1(4)$ \\
\hline II & $10(40)$ & $10(40)$ \\
\hline III & $11(44)$ & $14(56)$ \\
\hline IV & $4(16)$ & - \\
\hline
\end{tabular}

Tabela 2 Comorbidades entre os pacientes selecionados de acordo com a terapia designada

\begin{tabular}{|l|l|l|l|}
\hline Comorbidades & $\begin{array}{l}\text { PRP } \\
(\%)\end{array}$ & $\begin{array}{l}\text { Corticosteroide } \\
(\%)\end{array}$ & $\begin{array}{l}\text { Valor } \\
\text { de } \boldsymbol{p}\end{array}$ \\
\hline Obesidade & 76 & 88 & 0,46 \\
\hline Ausência de obesidade & 24 & 12 & 0,76 \\
\hline $\begin{array}{l}\text { Hipertensão arterial } \\
\text { sistêmica (HAS) }\end{array}$ & 64 & 68 & \\
\hline Ausência de HAS & 36 & 32 & 0,48 \\
\hline Diabetes & 24 & 16 & \\
\hline Ausência de diabetes & 76 & 84 & \\
\hline
\end{tabular}

Abreviação: PRP, plasma rico em plaquetas.
Com relação à evolução da pontuação na escala de WOMAC no período estudado, a média encontrada nos pacientes que usaram corticosteroides variou de 63,93 $(\Delta \mathrm{T}=0), 30,06(\Delta \mathrm{T}$ $=30)$, e 47,36 ( $\Delta \mathrm{T}=180)$ pontos. Comparativamente, as pontuações que usaram a mesma escala em pacientes cuja terapêutica foi o PRP tiveram valores médios de 73,94 $(\Delta \mathrm{T}=0)$, $21,96(\Delta \mathrm{T}=30)$, e $32,8(\Delta \mathrm{T}=180)$ pontos. Essa evolução da queixa álgica pode ser mais bem observada na figura $\mathbf{1}$.

Já em relação ao KSS, os valores médios obtidos no grupo de pacientes infiltrados com corticosteroides foram de 49,96 $(\Delta \mathrm{T}=0), 70,92(\Delta \mathrm{T}=30)$, e $66,92(\Delta \mathrm{T}=180)$ pontos. No grupo dos pacientes tratados com PRP foram encontrados os seguintes valores: $42,44(\Delta \mathrm{T}=0), 75,12(\Delta \mathrm{T}=30)$, e 75,36 $(\Delta \mathrm{T}=180)$ pontos. A evolução da funcionalidade dos pacientes pode ser mais bem observada na figura 2 .

De acordo com esses resultados, houve diferença estatisticamente significativa com favorecimento da terapia com o PRP em 180 dias em relação às escalas WOMAC e KSS. Por outro lado, não houve diferença entre os grupos de estudo nos primeiros 30 dias pós-tratamento. Também como demonstrado nas figuras 1 e 2, tanto o grupo corticoide quanto o grupo PRP evidenciaram melhoria significativa de acordo com as escalas de WOMAC e KSS nos 30 dias e 180 dias pós-infiltração.

Dessa maneira, os resultados obtidos ao se usar a terapia com PRP demonstrou-se mais duradoura, com recuperação funcional mais evidente e redução das queixas álgicas.

\section{Discussão}

O tratamento da osteoartrite de joelhos se inicia com uma conduta conservadora que envolve uma combinação de analgésicos, anti-inflamatórios não esteroides (AINEs), e fisioterapia. Conforme a complexidade do quadro ascende, outros métodos terapêuticos tornam-se importantes, como o uso de corticosteroides injetáveis, ácido hialurônico e, mais recentemente, infiltração de PRP. Os pacientes que usam AINEs e analgésicos apresentam melhor resposta terapêutica do que os que usam placebo, embora revisões de literatura considerem que evidências dos Aines no tratamento de osteoartrite de joelhos são limitadas a casos leves a moderados. ${ }^{25}$ A fisioterapia, comumente aplicada em pacientes portadores de osteoartrite de joelho, baseia-se na capacidade de manutenção de condicionamento físico, flexibilidade e fortalecimento do músculo quadríceps em auxílio ao tratamento medicamentoso. ${ }^{26}$ As injeções são usadas como terapias em pacientes não responsivos à medicação oral. ${ }^{11}$

Injeções com ácido hialurônico foram usadas por décadas, porém seu uso tem sido desencorajado devido ao alto risco de efeitos adversos e benefício irrelevante quando comparado com as injeções intra-articulares de corticosteroides. Além disso, o uso de ácido hialurônico não é subsidiado para o tratamento de acordo com as recomendações e diretrizes mais atuais. ${ }^{11,27}$ Dessa maneira, os corticosteroides injetáveis são amplamente usados no tratamento sintomático da osteoartrite de joelhos; entretanto, a sua eficácia parece ser limitada a 1 mês, e repetições do procedimento podem ser necessárias, o que pode ser feito até 3 vezes por ano na mesma articulação. ${ }^{12}$ 


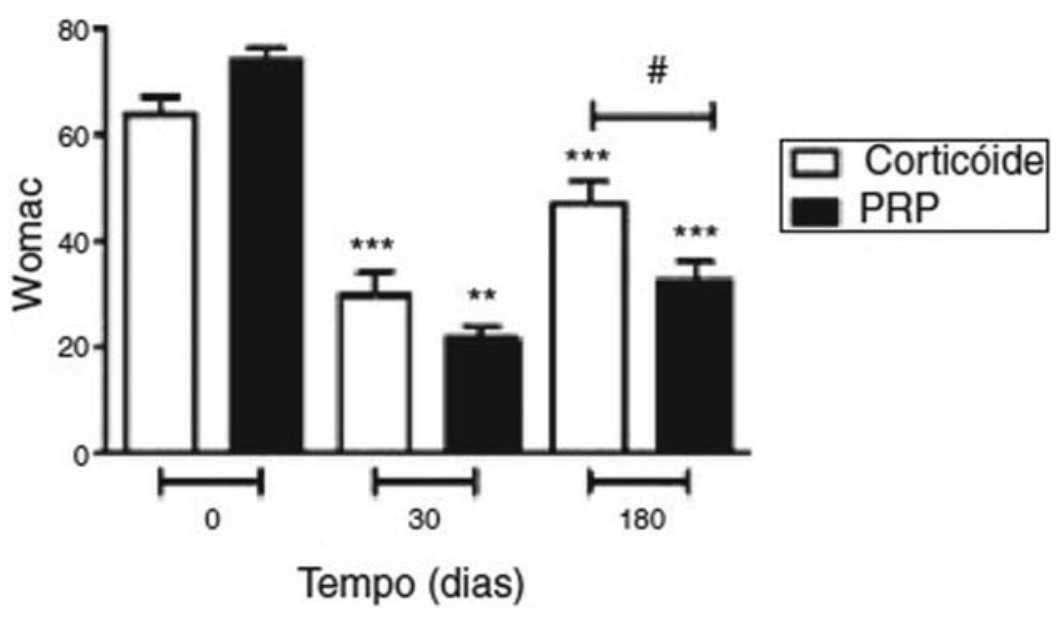

Fig. 1 Avaliação das respostas terapêuticas de acordo com a terapia com o questionário Western Ontario and MacMaster Universities Osteoarthritis Index aplicado em 0,30 , e 180 dias. $\left(\#=p<0,05 ;{ }^{* *}=p<0,01 ;{ }^{* * *}=p<0,001\right)$.

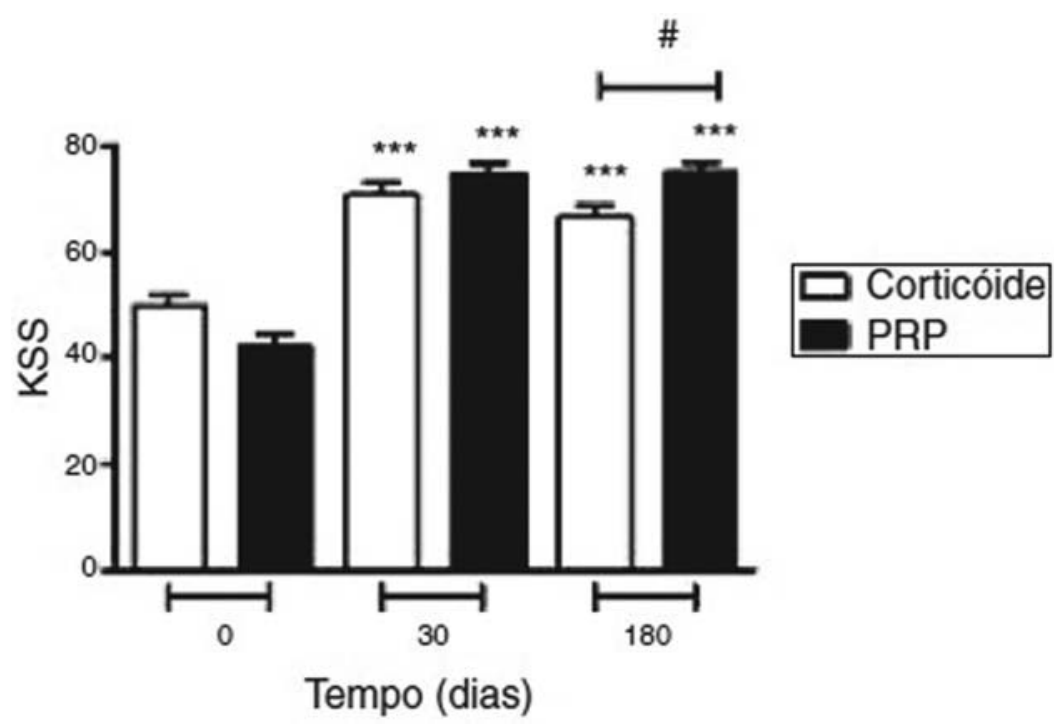

Fig. 2 Avaliação das respostas terapêuticas de acordo com a terapia com o questionário Knee Society Score aplicado em 0 , 30 , e 180 dias. $\left(\#=p<0,05 ;{ }^{* *}=p<0,01 ;{ }^{* * *}=p<0,001\right)$.

Dessa maneira, os dados encontrados no presente estudo corroboram o fato de que o efeito terapêutico dos corticosteroides é mais curto (até 30 dias), enquanto o perfil de melhora álgica prolongou-se até 180 dias após o uso de PRP.

O PRP é uma modalidade de tratamento em constante evolução que tem ganhado importância na atenção primária, reabilitação e medicina desportiva. ${ }^{15}$ PRP é uma solução concentrada de plaquetas preparada com sangue autólogo, o que o torna um tratamento seguro para o uso clínico. ${ }^{15}$ Estudos demonstraram que a administração da injeção intra-articular de PRP pode oferecer melhor qualidade de vida até mesmo após 1 ano de tratamento. ${ }^{16,28}$ O presente estudo avaliou que, em comparação com os corticosteroides, valores mais significativos quanto à melhoria clínica foram encontrados, especialmente em longo prazo da infiltração (180 dias). Ambos os pacientes obtiveram melhoria do quadro álgico e funcional em 30 dias e em 180 dias, porém os pacientes em uso da infiltração com PRP obtiveram melhoria mais importante do quadro álgico em números absolutos. Além disso, houve uma discreta redução da qualidade (5,6\%) no grupo de pacientes que usou corticosteroide intra-articular entre 30 e 180 dias, embora tenha se mantido estatisticamente mais significante do que no período pré-infiltrativo.

No presente estudo, verificamos a redução dos valores em escalas álgicas, como a WOMAC, e elevações das pontuações em escalas funcionais, como a KSS, mais evidentes em longo prazo (180 dias) ao usarmos o PRP. Embora ambas as terapias sejam eficazes e não haja significância entre elas, os resultados são importantes para aplicabilidade dessa modalidade terapêutica, uma vez que, por ser autólogo, emite menos riscos de efeitos colaterais do que a infiltração de corticosteroides com eficiência mais duradoura.

Segundo o estudo, $84 \%$ dos pacientes avaliados eram do idosas do sexo feminino.

Diabetes, hipertensão, cirurgias prévias, e obesidade têm sido relacionados a uma maior incidência de osteoartrite. ${ }^{5,29,30}$ Em nosso estudo, 66\% dos pacientes eram hipertensos e $82 \%$ eram obesos, corroborando assim os achados da literatura; 
entretanto, apenas $20 \%$ dos pacientes eram diabéticos e $2 \%$ haviam passado por cirurgias prévias.

Neste estudo, os fatores que elevam a incidência foram homogêneos entre os grupos quanto à escolha terapêutica, demonstraram fidedignidade da amostra e randomização prévia equivalente.

\section{Conclusão}

Os pacientes com artrose de joelho tratados tanto com infiltração com corticoide quanto com PRP reduziram as queixas álgicas e aumentaram a sua funcionalidade nos primeiros 30 e 180 dias pós-tratamento.

No entanto, de acordo com os dados apresentados nesse estudo, os índices de melhoria em longo prazo (com 180 dias) foram mais significativos no grupo tratado com PRP.

\section{Conflito de Interesses}

Os autores declaram não haver conflito de interesses.

\section{Referências}

1 Jordan KM, Arden NK, Doherty M, et al. Standing Committee for International Clinical Studies Including Therapeutic Trials ESCISIT. EULAR Recommendations 2003: an evidence based approach to the management of knee osteoarthritis: Report of a Task Force of the Standing Committee for International Clinical Studies Including Therapeutic Trials (ESCISIT). Ann Rheum Dis 2003;62 (12):1145-1155

2 Lawrence RC, Felson DT, Helmick CG, et al. National Arthritis Data Workgroup. Estimates of the prevalence of arthritis and other rheumatic conditions in the United States. Part II. Arthritis Rheum 2008;58(01):26-35

3 Rezende MU, De Campos GC. A osteoartrite é uma doenc,a mecânica ou inamatória? Rev Bras Ortop 2013;48(06):471-474

4 Scarpone M, Rabago D, Snell E, et al. Effectiveness of platelet-rich plasma injection for rotator cuff tendinopathy: a prospective open-label study. Glob Adv Health Med 2013;2(02):26-31

5 Pelletier JP, Martel-Pelletier J, Abramson SB. Osteoarthritis, an inflammatory disease: potential implication for the selection of new therapeutic targets. Arthritis Rheum 2001;44(06):1237-1247

6 Fitzgerald JB, Jin M, Grodzinsky AJ. Shear and compression differentially regulate clusters of functionally related temporal transcription patterns in cartilage tissue. J Biol Chem 2006;281 (34):24095-24103

7 Sarvimäki A, Stenbock-Hult B. Quality of life in old age described as a sense of well-being, meaning and value. J Adv Nurs 2000;32 (04):1025-1033

8 Clauw DJ, Witter J. Pain and rheumatology: thinking outside the joint. Arthritis Rheum 2009;60(02):321-324

9 Hameed F, Ihm J. Injectable medications for osteoarthritis. PM R 2012;4(5, Suppl):S75-S81

10 Evaniew N, Simunovic N, Karlsson J. Cochrane in CORR®: Viscosupplementation for the treatment of osteoarthritis of the knee. Clin Orthop Relat Res 2014;472(07):2028-2034

11 Bannuru RR, Natov NS, Obadan IE, Price LL, Schmid CH, McAlindon TE. Therapeutic trajectory of hyaluronic acid versus corticosteroids in the treatment of knee osteoarthritis: a systematic review and meta-analysis. Arthritis Rheum 2009;61(12): 1704-1711

12 Cheng OT, Souzdalnitski D, Vrooman B, Cheng J. Evidence-based knee injections for the management of arthritis. Pain Med 2012; 13(06):740-753
13 Nguyen RT, Borg-Stein J, McInnis K. Applications of platelet-rich plasma in musculoskeletal and sports medicine: an evidencebased approach. PM R 2011;3(03):226-250

14 Krogh TP, Bartels EM, Ellingsen T, et al. Comparative effectiveness of injection therapies in lateral epicondylitis: a systematic review and network meta-analysis of randomized controlled trials. Am J Sports Med 2013;41(06):1435-1446

15 Eppley BL, Woodell JE, Higgins J. Platelet quantification and growth factor analysis from platelet-rich plasma: implications for wound healing. Plast Reconstr Surg 2004;114(06):1502-1508

16 Patel S, Dhillon MS, Aggarwal S, Marwaha N, Jain A. Treatment with platelet-rich plasma is more effective than placebo for knee osteoarthritis: a prospective, double-blind, randomized trial. Am J Sports Med 2013;41(02):356-364

17 Sonnleitner D, Huemer P, Sullivan DY. A simplified technique for producing platelet-rich plasma and platelet concentrate for intraoral bone grafting techniques: a technical note. Int J Oral Maxillofac Implants 2000;15(06):879-882

18 Messora MR, Nagata MJ, Furlaneto FA, et al. Análise da eficiência do protocolo de dupla centrifugac,ão para o preparo do plasma rico em plaquetas (PRP) - estudo experimental em coelhos. RSBO Rev Sul-Bras Odontol. 2009;6(03):291-296

19 Schiphof D, Boers M, Bierma-Zeinstra SMA. Differences in descriptions of Kellgren and Lawrence grades of knee osteoarthritis. Ann Rheum Dis 2008;67(07):1034-1036

20 Silva AL, Demange MK, Gobbi RG, da Silva TF, Pécora JR, Croci AT. Translation and Validation of the Knee Society Score - KSS for Brazilian Portuguese. Acta Ortop Bras 2012;20(01):25-30

21 Bellamy N, Buchanan WW, Goldsmith CH, Campbell J, Stitt LW. Validation study of WOMAC: a health status instrument for measuring clinically important patient relevant outcomes to antirheumatic drug therapy in patients with osteoarthritis of the hip or knee. J Rheumatol 1988;15(12):1833-1840

22 Dinjens RN, Senden R, Heyligers IC, Grimm B. Clinimetric quality of the new 2011 Knee Society score: high validity, low completion rate. Knee 2014;21(03):647-654

23 Oliveira AM, Peccin MS, Silva KN, Teixeira LE, Trevisani VF. Impact of exercise on the functional capacity and pain of patients with knee osteoarthritis: a randomized clinical trial. Rev Bras Reumatol 2012;52(06):876-882

24 Hmamouchi I, Allali F, Tahiri L, et al. Clinically important improvement in the WOMAC and predictor factors for response to nonspecific non-steroidal anti-inflammatory drugs in osteoarthritic patients: a prospective study. BMC Res Notes 2012;5:58

25 Eccles M, Freemantle N, Mason J; The North of England NonSteroidal Anti-Inflammatory Drug Guideline Development Group. North of England evidence based guideline development project: summary guideline for non-steroidal anti-inflammatory drugs versus basic analgesia in treating the pain of degenerative arthritis. BMJ 1998;317(7157):526-530

26 Roddy E, Zhang W, Doherty M. Aerobic walking or strengthening exercise for osteoarthritis of the knee? A systematic review. Ann Rheum Dis 2005;64(04):544-548

27 Mei-Dan O, Carmont MR, Laver L, Mann G, Maffulli N, Nyska M. Platelet-rich plasma or hyaluronate in the management of osteochondral lesions of the talus. Am J Sports Med 2012;40(03):534-541

28 Leitner GC, Gruber R, Neumüller J, et al. Platelet content and growth factor release in platelet-rich plasma: a comparison of four different systems. Vox Sang 2006;91(02):135-139

29 Mohammadi F, Taghizadeh S, Ghaffarinejad F, Khorrami M, Sobhani S. Proprioception, dynamic balance and maximal quadriceps strength in females with knee osteoarthritis and normal control subjects. Int J Rheum Dis 2008;11(01):39-44

30 Oliveira NC, Vatri S, Alfieri FM. Comparação dos efeitos de exercícios resistidos versus cinesioterapia na osteoartrite de joelho. Rev Acta Fisiátr. 2016;23(01):7-11 